\title{
Design of Demand Response Analysis Control Architecture Based on Multi-Agent Technology
}

\author{
Lei Ting \\ Electric Power Research Institute \\ State Grid Shanghai Municipal Electric \\ Power Company \\ Shanghai, China \\ e-mail: 1t1986130@163.com
}

\author{
Xie Yingxin, Wang Fengyu, Liu \\ Yin, Dong Gang \\ Beijing GuoDianTong Network \\ Technology Co. Ltd., \\ Beijing, China \\ e-mail: liuyin_1989@163.com
}

\author{
Fang Chen \\ Electric Power Research Institute \\ State Grid Shanghai Municipal Electric \\ Power Company \\ Shanghai, China \\ e-mail: 9970993@qq.com
}

\begin{abstract}
Demand response (DR) is one of the best applications to practice the idea of coordinated interaction between smart grid and the electrical loads. In order to support the development of DR in the smart grid, this paper presents and designs a DR analysis control architecture based on Multi-Agent (MA) technology according to the organization of region, user and device. Firstly, the three-layer distributed DR analysis control architecture is described, and the main features of the architecture are listed. Secondly, the implementation method for the agent at each layer is given in detail, including the functional components and working principles. Finally, a full text summary is concluded. With the help of this DR control Architecture we can conduct different levels of DR control by a hierarchical control model, which can be adapted to the distributed characteristics and flexible control modes of the diverse loads very well, and achieve the purpose of enhancing the balance capabilities of regional load and the overall energy efficiency finally.
\end{abstract}

Keywords-Smart grid; Demand response; Multi-Agent; Hierarchical control

\section{INTRODUCTION}

In recent years, with the contradiction between electricity supply and demand, as well as more serious pressure of energy conservation and emission reduction, Traditional power system, which adjusts power supply mode according to the load curve, exposes its drawbacks increasingly. Thinking about how to achieve a dynamic balance between supply and demand by "Grid -Load" coordination interaction from the perspective of the user side is a good idea. And this idea can improve the power grid peak difference and resolve resource and environmental pressure, and it will become inevitable development direction of the grid in future ${ }^{[1-2]}$. As one of the best applications to practice the idea of smart grid coordinated interaction between the smart grid and the electrical loads, demand response (DR) is a series of actions taken in order to balance power supply and demand, and it can assist the grid to solve issues about the power grid reliability and peak demand management without increasing the cost of electricity and capacity ${ }^{[3-5]}$. Specifically, the basic forms of conventional DR include price-based DR and incentive-based DR. In the price-based DR, consumers reduce or shift their peak demand usage in response to price signals, while in the incentive-based DR, consumers interrupt their power usage voluntarily for financial incentives. With further advancement of smart grid construction, diverse loads including energy storage devices, electric vehicles, smart appliances, smart buildings and so on, are widely used in the demand side. Under this situation, the realization process of DR control is facing a new challenge. Due to the current domestic demand side management is still in the stage that the power grid dominates and consumers passively accept, load control belongs to the traditional centralized control. In the face of the diverse loads' distributed characteristics and flexible control modes, the lack of DR control scheme which consumers can participate actively is a serious problem.

Multi-Agent (MA) is a technology which can be able to intelligently and flexibly respond to changes of the working conditions and the needs of peripheral process. It has a wide application throughout the whole power system ${ }^{[6-7]}$. The paper [8] used this technology to solve the problem of selfhealing control of distributed power grid, the results showed that it realized the function of fast recovery after the grid fault. The paper [9] proposed a micro power grid control framework based on MA technology. Specifically, a MA based control system is established which had three layers including the superior power grid agent, micro power grid agent and components agent, providing a reliable guarantee for the efficient and stable operation of the micro-grid. However, MA has not yet been applied in DR control currently. Therefore, this paper presents a MA-based DR analysis control architecture that fully takes the price-based DR and incentive-based DR into account. This architecture adopts the idea of centralized - decentralized and hierarchical-classificatory control, which can well be adapted to the distributed characteristics and flexible control modes of the diverse loads.

\section{CONTROL FRAMEWORK ANALYSIS OF DR}

\section{A. Framework Constitution}

The MA-based DR analysis control architecture is shown in Figure 1. It is divided into three control structures, namely Regional Layer Agent (RLA), User Layer Agent (ULA) and 
Device Layer Agent (DLA). RLA is primarily responsible for coordinating scheduling among user agents in ULA. After receiving the task issued from the superior grid agent, RLA makes an appropriate coordinating scheduling plan for ULA by obtaining historical load data, the running state of power grid, load curtailment instructions and other informations of ULA, and then RLA sends this plan to ULA. In this architecture, RLA can be acted by the regional energy management system.

As a mid-management system, ULA does not only exchange information to ULA, but also needs to issue execute instructions to DLA. ULA takes the initiative to declare participation scheme of DR to RLA and waits the execute instructions from RLA. Until receiving instructions, ULA combines a lot of informations, such as the running time, the important grade of the power load and the power supply capacity of distributed power, energy storage devices and other interactive loads etc., to make a execute scheme and releases to DLA. According to information obtained, DLA makes a decision and changes the operating status of the device.

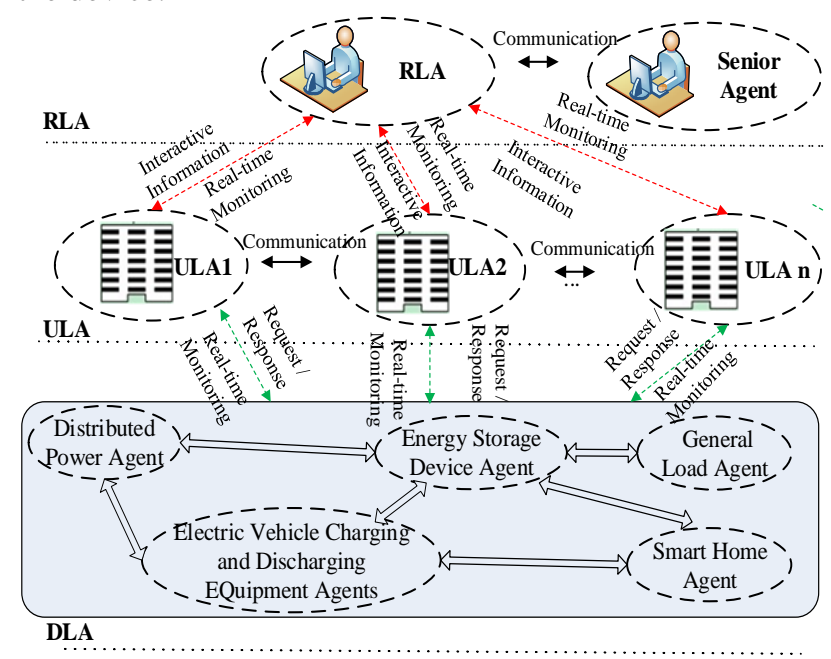

Figure 1. Demand response analysis control architecture

\section{B. Framework Features}

The MA-based DR analysis control architecture is mainly possessed the characteristics of MA technology, such as autonomy, initiative, and collaboration and consultation, as well as considers the two-way interaction between the grid and the users in the implementation process of DR. In general, the architecture has the following advantages.

- Incentive-based DR covers three implementation methods, that is demand side bidding, interruptible load control and direct load control. Through pushing interaction model suggested by RLA to the user and declaring DR participation scheme initiatively by ULA the consumers changes the role from price taker into price provider, which can ensure the enthusiasm of participation and the effectiveness of demand side management.

- By taking delamination, hierarchical control method, the relationship between each agent is not only coordinated but also independent. The entire control framework has good robustness and flexibility. The number of running agent is unlimited at the same time. When an agent joins or exits, other agents will quickly adjust according to the logical relationship coordination control strategy.

- MA technology itself has a good centralizeddecentralized characteristics. The control framework is a distributed coordination control behavior, which allows a certain degree of autonomy and intelligence for agents, by distributing power of control to each agent member. Agent members change their own electricity behaviors according to the load operation mode of themselves, the current operating status of the power grid and the tariff policy and other informations. The MA-based DR analysis control architecture overcomes the limitations of centralized load management effectively.

- Fully considering the running needs of grid side and the will of user side, ULA can choose DR participation mode and compensation rates flexibly by enhancing the "Grid-Load" interaction. ULA changes their participation approaches from passive acceptance to active participation, while RLA selects interactive load preferentially whose peaking costs are lower, consequently, demand matching and mutual benefit win between supply and demand are realized.

\section{AGENTS IMPLEMENT}

In the overall architecture, the RLA and ULA are architecture analysis and decision-makers, and the DLA plays a part of direct executor. As realization of the DLA is relatively simple, there are no details in this paper. This chapter mainly describes composition modules and implementation principles of the RLA and ULA.

\section{A. Implement of RLA}

As shown in Figure 2, RLA consists of six modules that are database storage, intelligent computing, information exchange, parameter setting, statistical analysis and monitoring show. Database storage module is mainly used to save the contents, including the load reduction information, the control decision-making model, controllable load resource information submitted by users, the power information and so on. Intelligent computing module analyzes and generates load control plan intelligently by multi-objective optimization algorithm. Information exchange module provides an interface for RLA and ULA, and is responsible for sending plan and uploading electricity information. Parameter setting module is a way to achieve human-computer interaction, and its main task is to set optimization objectives and constraints and other information about control decision model. Statistical analysis module counts and analyzes the electricity information uploaded from ULA. Monitoring show module displays the results of load control simulation and actual implementation. 


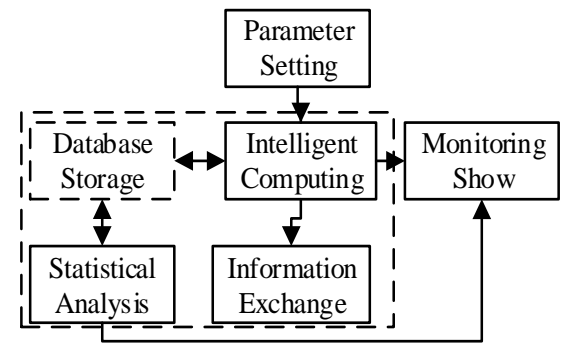

Figure 2. The main function modules of RLA

On the basis of the functional modules of RLA, the way to realize DR control process needs three steps: firstly, a controllable load resource library based on participation information reported by ULA is created. Where, the unit of controllable load is a user. Secondly, a DR plan is made according to the command from higher level agent or the own needs of RLA and pushed to the ULA. Finally, a statistical analysis for implementation effectiveness of ULA's feedback is established and the related information in controllable load resource library is updated accordingly. The specific working principle is shown in Figure 3.

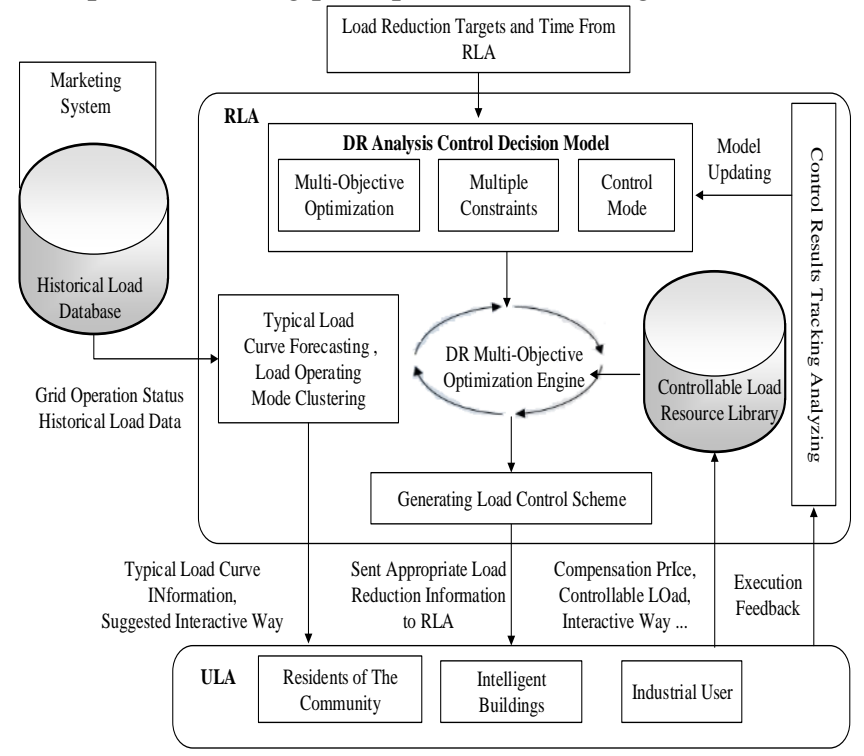

Figure3. Implementation structure diagram of RLA

1) Creating controllable load resource library:Creating controllable load resource library is the key to achieve DR analysis control. It covers not only the way in which users interact with DR participation, but also encourages users to participate in DR, including the interaction of the price mechanism. In the process of creating controllable load resource library, in order to avoid blindness of DR submitted by ULA who participates in the scheme, it uses historical load information to form load curve forecasting, clustering and decision- analyzing for diverse user in ULA, extracts a typical load curves which can be the most representative of user load characteristics and makes a classification of these load curves, and provides a targeted suitable DR interaction suggestions for users who have same characteristics of electricity combining the user's production and living nature. Where, DR interaction suggestions may include interactive mode, such as load interruption, load transfer and so on. All of those can auxiliary support the implementation of demand-side response analysis control. On the basis of interaction informations recommended by RLA,ULA develops and reports DR participation scheme, which may include load interaction mode, the amount of load control, load compensation price, controllable time and other contents, in line with the its own circumstances demand. This mudule will take the user who participates in DR into the controllable load resource library for future calls.

2) Generating and transmitting DR control scheme: Combining power load reduction information and regulation time requirements, this module establishes DR analysis control decision model based on certain optimization goals(such as the minimum revenue of grid loss, the smallest users of electricity affected area, the highest reliability of scheme implementation, etc.) and some constraints. It automatically generates DR load control scheme which meets the objectives through multi-objective optimization engine of DR. After the generating scheme, the scheme is confirmed by a operator with authority and then will be sended to corresponding ULA.Then the next is ULA's work.

3) Analyzing the effect of DR load control: This module tracks and analyzes the effect of DR load control scheme according to the implementation feedback from ULA. So as to provide amended information for control the decisionmaking model to the DR analysis.

\section{B. Implement of ULA}

As the following Figure 4 shows, the ULA is composed of six modules: database storage, DR analysis control, DR plan generation, information exchange, parameter setting, and monitoring show.

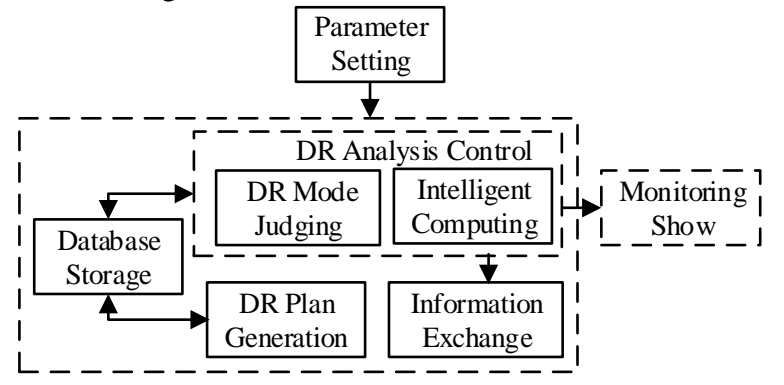

Figure 4. The main function modules of ULA

Database storage module is mainly used to save the contents, including controllable load resources information, electricity price and incentive information, operation information of power grid, control decision-making model, and load shedding command information from RLA, and so on. DR analysis control module supports both incentivebased DR and price-based DR, so this module is composed of DR mode judging unit and intelligent computing unit, in 
order to generate DR load control scheme for users. DR plan generation module makes DR participating scheme declared to RLA according to their own situation. The functions of parameter setting module, information exchange module and monitoring show module are same with RLA, but must be designed depending on the specific conditions.

ULA realizes DR control process with three steps. The concrete working principle is as follows in Figure 5.

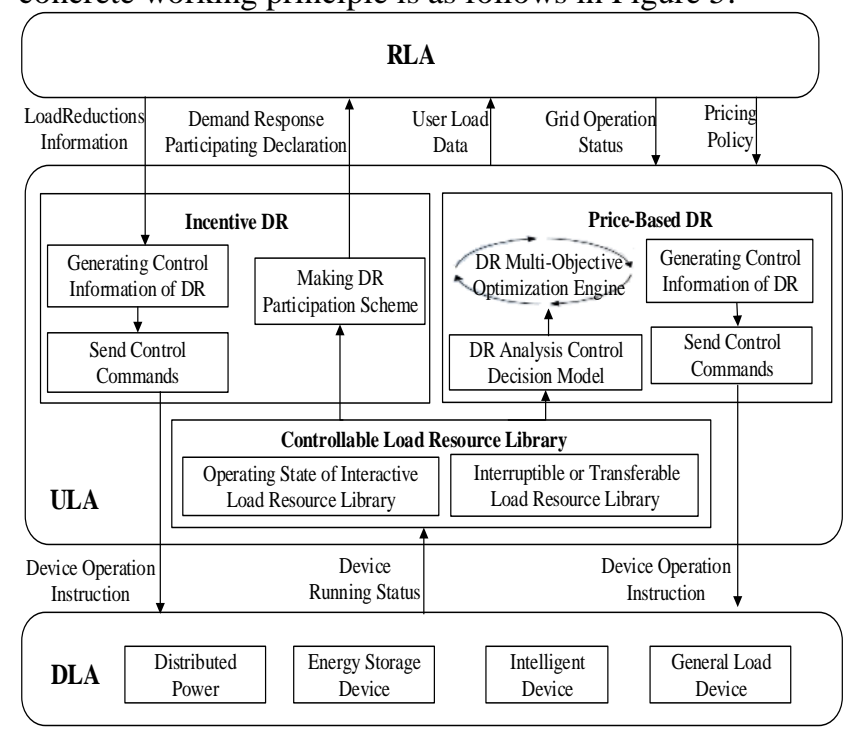

Figure 5. Implementation structure diagram of ULA

1) Establishing controllable load resource library: As mentioned above,ULA and RLA are different in category, definition and information about loas are not the same. Controllable load resources here are mainly aimed at the bottom of the load devices. According to the interaction mode between grid and load, controllable load resource library is divied into operating state of interactive load resource library and interruptible or transferable load resource library. This module is primarily responsible for maintaining information about the device,including load current operating status, rated capacity, important level, the normal boot and shutdown times, minimum continuous running time, maximum interrupt time, the maximum times of the controlled and so on.

2) Generating and transmitting DR control scheme: If one user agent finds that his load operating mode has greater flexibility and adjustability, and wants to adjust conduct electricity appropriatly to obtain financial compensation, then he cantake the initiative to declare incentive-based DR plan.ULA needs to statistictotal capacity of controllable load,demand interactive mode, the duration of regulation and the compensation price etc., accroding the information of controllable load resource library. On basis of this, this module develops the best participation plan which can obtian the best economic interests, and then reports this plan to RLA. When RLA has peaking demand and ULA also receives the reductions instruction from RLA, this module combines controllable load operation information to generate action commandsof DLAand sends these commands to DLA. In addition, it can also combine with the power grid operating conditions, inculdingthe current operating status, price policy and diversification of the load,to establishDR analysis control decision model.The model must meet certain control objectives (such as the least affect user comfort, the lowest cost of electricity and other objectives considering needs) and some constraint conditions. Price-based DR and incentive DR should be complementary to each other in the whole architecture.

3) Uploading load data. ULA should push the relevant load data to RLA periodically, which assists RLA in tracing and analyzing executive effect of DR. And on this basis, RLA allots corresponding economic compensation to ULA.

\section{CONCLUSION}

This paper designs a DR analysis control architecture. The architecture relates to price-based DR and incentivebased DR and gives full consideration to common interests between grid and user. It succeeds in realizing DR analysis and control by adopting MA technology which has the property of centralized - decentralized, hierarchicalclassificatory control mode. Overall system architecture describes the mutual operating mechanism between the three layers, namely RLA, ULA and DLA. Advantages of this architecture are represented as well as structure. Then this paper introduces sub-function contained within each layer agent and also describes associated implementation and work flow. The MA-based DR analysis control architecture presented in this paper is built to a modular design with data mining technology and has high scalability and wide application. It provides a firm institutional guarantee for improving overall efficiency and widespread using of DR in smart grid.

\section{ACKNOWLEDGMENT}

This work was financially supported in part by National Key Technology R\&D Program (No.2013BAA01B04), Science and Technology Commission of Shanghai Municipality (No.11dz1210401), and the Science and technology projects of State Grid Corporation of China (No.520940120036).

\section{REFERENCES}

[1] Yang Dechang, Li Yong, C. Rehtanz, Liu Zehong and Luo Longfu "Study on The Structure and The Development Planning of Smart Grid in China". Power System Technology, 2009, 33(20).

[2] Yu Yixin, and Luan Wenpeng. "Smart Grid Review". Proceedings of the CSEE, 2009, 29(34).

[3] M. H. Albadi, and E. F. Eisaadany. "A Summary of Demand Response in Electricity Markets”. Electric Power Systems Research. 2008.

[4] S. Valero, M. Ortiz, and C. Senabre. "Methods for Customer and Demand Response Policies Selection in New Electricity Markets". IET Generation, Transmission \& Distribution. 2007. 
[5] Liu Hongiin, Yuan Bin, and Dai Hongwei. "Framework Design of a General-purpose Power Market Simulator Based on Multi-agent Technology". Power Engineering Summer Meeting. 2001.

[6] Shu Hongchun, Tang Lan, and Dong jun."A Survey on Application of Multi-Agent technology in Power System". Power System Technology, 2005, 29(6):28-31.

[7] John A. Hossack, Judith Menal, and D.J Stephen. "A Multi-agent Rrchitecture for Protection Engineering Diagnostic Assistance”. IEEE Transactions on Power Systems.

[8] LIU Xindong, LI Weihua, ZHU Yong, WANG Dedao, and FU Xiao. "Research on Self-healing Control of Distributed Networks Based on Multi-Agent Technology". Power System Protection and Control. 2012, 17:116-120.
[9] Xu Dong, and Ding Wenfang. “ A Study of Micro-grid Control Based on Multi-Agent ".Journal of Hubei University of Technology, 2011, 03:70-73.

[10] Han Jiawei, and Micheline Kamber. "Data Mining Concepts and Techniques". Beijing:China Machine Press,2006:30-46.

[11] Cheng Qiyun. "Research on Power Short-Term Load Forecasting Model and Method Based on Data Mining".Chongqing": Chongqing University,2004.

[12] K. P. Soman, S. Diwakar, and V. Ajay. "Insight Into Data Mining Theory and Practice".India:Prentice Hall,2006:234.

[13] M. Ehta, R. Agrawal, and J. Rissanen. "SLIQ: A Fast Scalable Classifier for Data Mining".In Proceeding 1996 International conference Extending Database Technology (EDBT'96). Avignon. France.Mar.1996. 